\title{
Probabilistic fatigue analysis of existing steel structure
}

\author{
Martin Krejsa ${ }^{1, *}$, Jiři Brožovský ${ }^{1}$, Petr Lehner ${ }^{1}$, Stanislav Seitl ${ }^{2,3}$, Zdeněk Kala ${ }^{3}$, and \\ Vlastimil Krejsa ${ }^{1}$ \\ ${ }^{1}$ VSB-Technical University of Ostrava, Faculty of Civil Engineering, Department of Structural \\ Mechanics, Ludvika Podeste 1875/17, 70833 Ostrava-Poruba, Czech Republic \\ ${ }^{2}$ Institute of Physics of Materials of the Academy of Sciences of the Czech Republic, Zizkova 22, \\ 61662 Brno, Czech Republic \\ ${ }^{3}$ Brno University of Technology, Faculty of Civil Engineering, Institute of Structural Mechanics, \\ Veveri 331/95, 60200 Brno, Czech Republic
}

\begin{abstract}
The paper deals with evaluation of possible fatigue damage of existing steel crane structure. It is possible to use the some traditional methods to solve the problem - a concept of fatigue S-N curves (which are mainly used for designing of a new structures) or the procedures based on linear fracture mechanics utilizing the Paris-Erdogan's law (which are suitable for estimation of remaining lifetime). For the resistance assessment the relevant data of load effects caused by cyclically loaded structure operation are necessary for both concepts. For the prediction of fatigue damage over time, calibration functions for short edge cracks were derived based on the results of the experiment, and the acceptable size of the fatigue crack in damaged structural component under analysis was determined. Using the derived relationships, a stochastic analysis of the selected element was performed and the results are discussed.
\end{abstract}

\section{Introduction}

Fatigue phenomenon is one of the main factors influencing the life of steel structures and bridges subjected to cyclic loading. This paper focuses on the probabilistic analysis of fatigue damage of the supporting structure of the crane runway (see Fig. 1) serving the steel warehouse operation of the Vitkovice Machinery Group, Czech Republic, which also belong to the structures subjected to repeat loading.

The assessment of fatigue life and, in particular, the prediction of residual service life in the existing buildings are a significant and current engineering problem [1]. Owing to the ageing of the existing infrastructure on a worldwide scale and the lack of codes for the continued safety management of structures during their lifetime, it is necessary to develop mathematical tools to evaluate their structural reliability over a time interval, and considering the reduction in their structural capacities. A number of studies have been conducted for the stochastic estimation of reliability and the subsequent prediction of the life of various carrying capacity elements and constructions, e.g. [2-7]. However, a valid calculation

* Corresponding author: martin.krejsa@vsb.cz 
methodology based on probabilistic modelling that would be directly applicable to the existing load-bearing structures is non-existent [8].

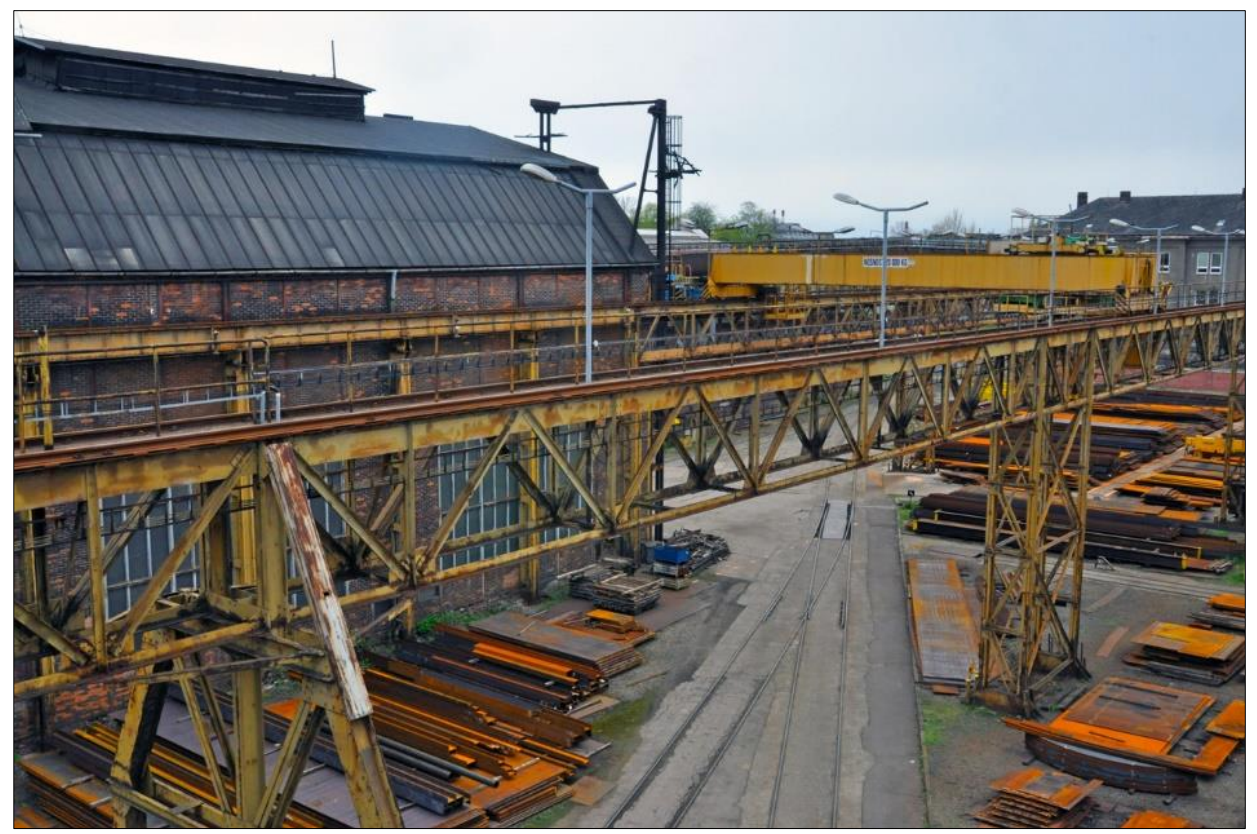

Fig. 1. Analysed steel truss structure supporting an overhead crane.

Numerous numerical methods, mostly based on the finite element method - FEM [9-12], have been developed to aid in the understanding of the behavior of the fatigue phenomena. The essential tools for these calculations are provided by fracture mechanics [13] and the reliability theory $[14,15]$. Some of approaches used for the fatigue crack prediction are based on stochastic methods $[16,17]$. Insight into the stochastic interactions among random factors (load, geometric and material characteristics) affecting the reliability of steel bridges is namely essential and crucial to understanding the progress of failure probability of steel structures over time. Moreover, due to the presence of significant uncertainties associated with crack initiation and propagation, inspection, monitoring and/or repair actions planning [18] should be performed and applied to prevent sudden failures of damaged structural components and their associated consequences.

\section{Fatigue crack propagation}

Reliability of the bearing structure has been significantly influenced by degradation resulting, in particular, from the fatigue of the basic materials. When investigating the propagation, the fatigue crack that deteriorates a certain area of the structure component is described with one dimension only - fatigue crack length $a$. In order to describe the propagation of the crack, the linear elastic fracture mechanics is typically used [13]. This method uses Paris-Erdogan's law [19] and defines relation between propagation rate of the crack size $a$, and range of the stress rate coefficient, $\Delta K$, in the tip of the crack:

$$
\frac{\mathrm{d} a}{\mathrm{~d} N}=C \cdot(\Delta K)^{m}
$$


where $C, m$ are material constants, that are determined experimentally, $N$ is the number of loading cycles and $\Delta K$ is range of the stress intensity factor in front of the crack tip. Range of the stress intensity factor is defined as follow:

$$
\Delta K=\Delta \sigma \cdot \sqrt{\pi \cdot a} \cdot F_{(a)},
$$

where $\Delta \sigma$ is constant stress range and $F_{(a)}$ is the calibration function which represents propagation of the crack (for instance, from the edge or surface) and various boundary conditions.

Three sizes are important for the description of the characteristics of the propagation of fatigue cracks. The fatigue crack will propagate in a stable way only if the initial crack $a_{0}$ exists in the place where the stress is concentrated. Existence of the initiation cracks during the propagation should be revealed, along the detectable length of the crack $a_{d}$, e.g., during inspections. The crack propagates in a stable way until it reaches the third important size acceptable length of the crack $a_{a c}$, which is a limit for the required reliability. When using Eq. (1), the condition for the acceptable crack length, $a_{a c}$, is:

$$
N=\frac{1}{C} \cdot \int_{a_{0}}^{a_{a c}} \frac{\mathrm{d} a}{(\Delta K)^{m}}>N_{t o t},
$$

where $N$ is the number of cycles needed to increase the crack from the initiation size $a_{0}$ to the acceptable crack size $a_{a c}$, and $N_{\text {tot }}$ is the number of cycles throughout the service life.

The main assumption is that the primary design should take into account the effects of the extreme loading and the fatigue resistance should be assessed. The probabilistic methods should be used for the investigation of the propagation rate of the fatigue crack until the acceptable size is reached because the input variables include uncertainties and reliability should be taken into account. The equation for the propagation of the crack size (1) needs to be modified for this purpose. After the change of the number of cycles from $N_{1}$ to $N_{2}$, the crack will propagate from the length $a_{1}$ to $a_{2}$. Having modified Eq. (1) and using Eq. (2), the following formula will be achieved:

$$
\int_{a_{1}}^{a_{2}} \frac{\mathrm{d} a}{(\sqrt{\pi \cdot a} \cdot F(a))^{m}}=\int_{N_{1}}^{N_{2}} C \cdot(\Delta \sigma)^{m} \mathrm{~d} N
$$

If the length of the crack $a_{1}$ equals to the initial length $a_{0}$ (this is the assumed size of the initiation crack in the probabilistic approach) and if $a_{2}$ equals to the final acceptable crack length $a_{a c}$, then the left-hand side of the Eq. (4) can be regarded as the resistance of the structure $R_{\left(a_{a c}\right)}$ :

$$
R_{\left(a_{a c}\right)}=\int_{a_{0}}^{a_{a c}} \frac{\mathrm{d} a}{\left(\sqrt{\pi \cdot a} \cdot F_{(a)}\right)^{m}}
$$

Similarly, it is possible to define the cumulated effect of loads (random variable effects of the extreme load) that is equal to the right side of Eq. (4):

$$
E_{(N)}=\int_{N_{0}}^{N} C \cdot(\Delta \sigma)^{m} \mathrm{~d} N=C \cdot(\Delta \sigma)^{m} \cdot\left(N-N_{0}\right),
$$

where $N$ is the total number of oscillations $\Delta \sigma$ for the change of the length from $a_{0}$ to $a_{a c}$, and $N_{0}$ is the number of oscillations in the time of initialization of the fatigue crack (typically, the number of oscillations is zero). 


\section{Stochastic reliability assessment}

As with other probabilistic methods, a safety margin $Z$ can be defined for reliability assessment:

$$
Z_{(\mathbf{X})}=R_{\left(a_{a c}\right)}-E_{(N)}
$$

where $\mathrm{X}$ is a vector of random physical properties such as mechanical properties, geometry of the structure, load effects and dimensions of the fatigue crack. The analysis of the safety margin $Z$ gives a failure probability $P_{f}$, which is equal to:

$$
P_{f}=P\left(R_{\left(a_{a c}\right)}<E_{(N)}\right)=P\left(R_{\left(a_{a c}\right)}-E_{(N)}<0\right)=P\left(Z_{(\mathbf{X})}<0\right) .
$$

\section{Application}

Described probabilistic analysis of fatigue damage was applied to a nearly 100 -year old exterior riveted crane support truss (see Fig. 1) focusing on the most susceptible element in terms of fatigue damage - a diagonal formed by a pair of profiles L90x10 in the middle of the truss structure. The study follows the article [20] where the fatigue resistance and the residual life estimation of this structure was analyzed using numerical modelling in ANSYS software based on rainflow counting and the Palmgren-Miner rule.

The input quantities were determined deterministically (geometric parameters, $m=3, C=2.2 \cdot 10^{-13} \mathrm{MPa}^{m} \mathrm{~m}^{m / 2+1}$ ) or stochastically using parametric probability distributions (e.g. yield stress of old steel obtained by laboratory experiment, published in [21]; axial force as time-dependent load effect based on the operation of the overhead crane generated by Monte Carlo method, published in [20]; initial size of the crack $a_{0}$ according to [22]). Calibration functions $F_{(a)}$ describing the way of fatigue crack propagation for different types of loads in Eq. (5) were determined experimentally were published in [23, 24]. The required reliability was described by the reliability index $\beta=2$ which corresponded to the target probability $P_{d}=0.02277$.

The described methodology for stochastic modelling of fatigue phenomena has been

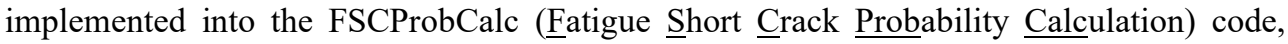
which allows probabilistic calculation to be performed efficiently in a user-friendly environment. Using the program the probability evaluation of construction reliability focused on fatigue damage can be carried out upon exact definition of fatigue crack admissible size and definition of occurrence probability of three basic facts related with spreading of fatigue cracks which lead to calculation of probability defect for each operational year of the solved construction. At setting of the reliability required rate also time of the first inspection of the construction can be set which will be focussed on fatigue damage and using the conditioned probability also times of the following inspection checks. Figs. 1 to 6 show the program's desktop with calculated results.

The Fig. 2 presents a histogram stress range $\Delta \sigma$ in Eq. (6), calculated according to e.g. [24]. Fig. 3 shows the resulting histogram of structural resistance $R_{\left(a_{a c}\right)}$ calculated according Eq. (5). Fig. 4 contains the accumulated load effect $E_{(N)}$ for 277 years of structural operation determined by Eq. (6). Both variables are needed to define the safety margin $Z_{(\mathbf{X})}$ according Eq. (7), see Fig. 5, and probability of failure $P_{f}$ estimation in Eq. (8) for the relevant year of operation of the structure under assessment, see Fig. 6. 


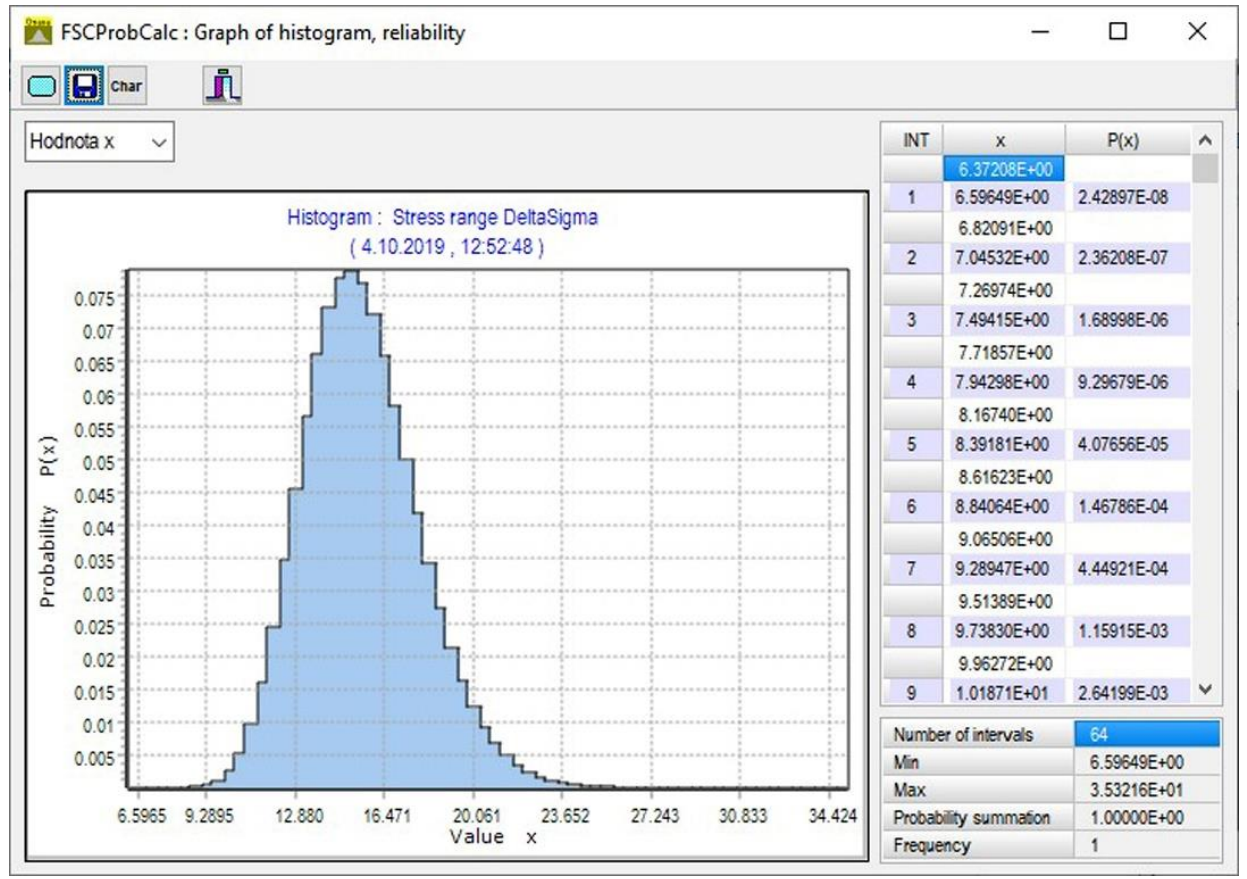

Fig. 2. FSCProbCalc desktop: Calculated histogram of stress range $\Delta \sigma$.

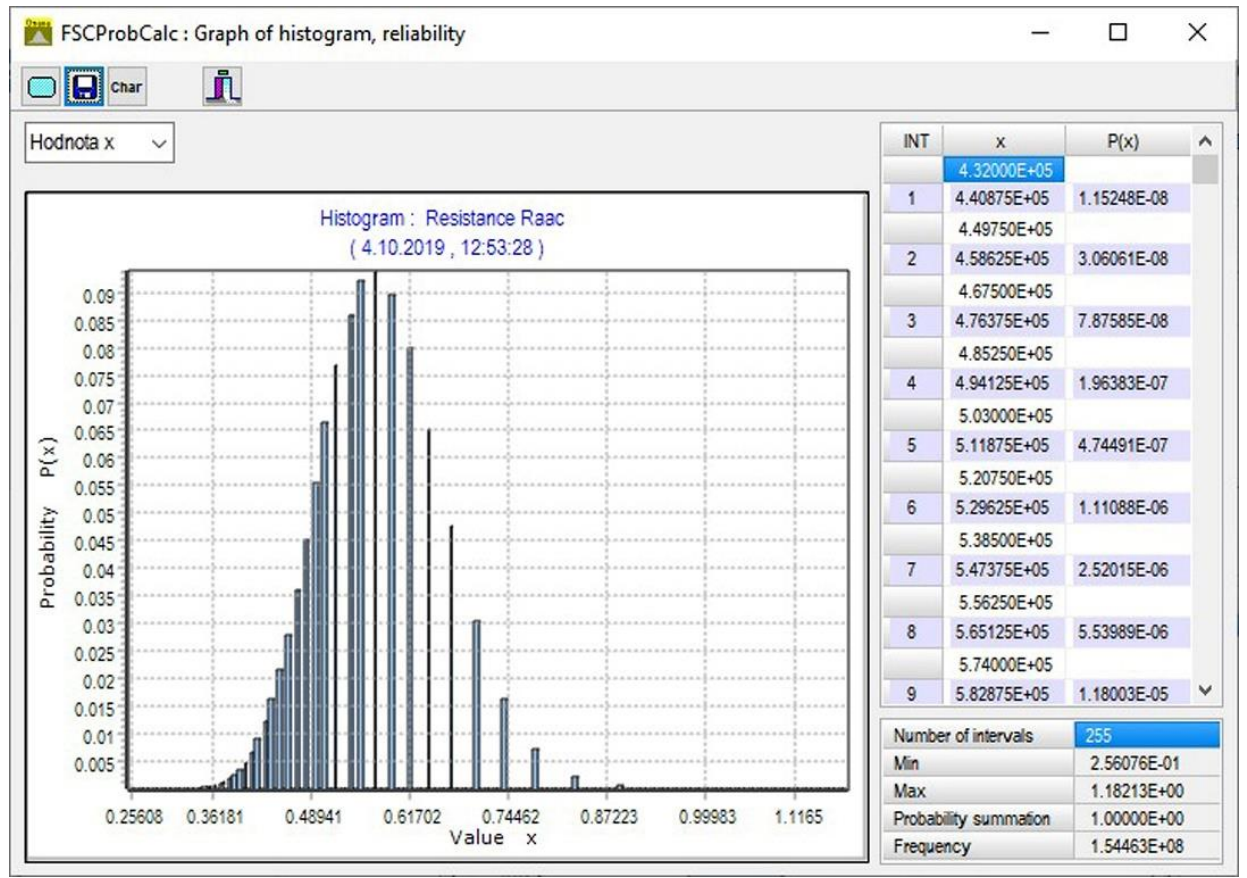

Fig. 3. FSCProbCalc desktop: Calculated histogram of resistance $R_{\left(a_{a c}\right)}$. 


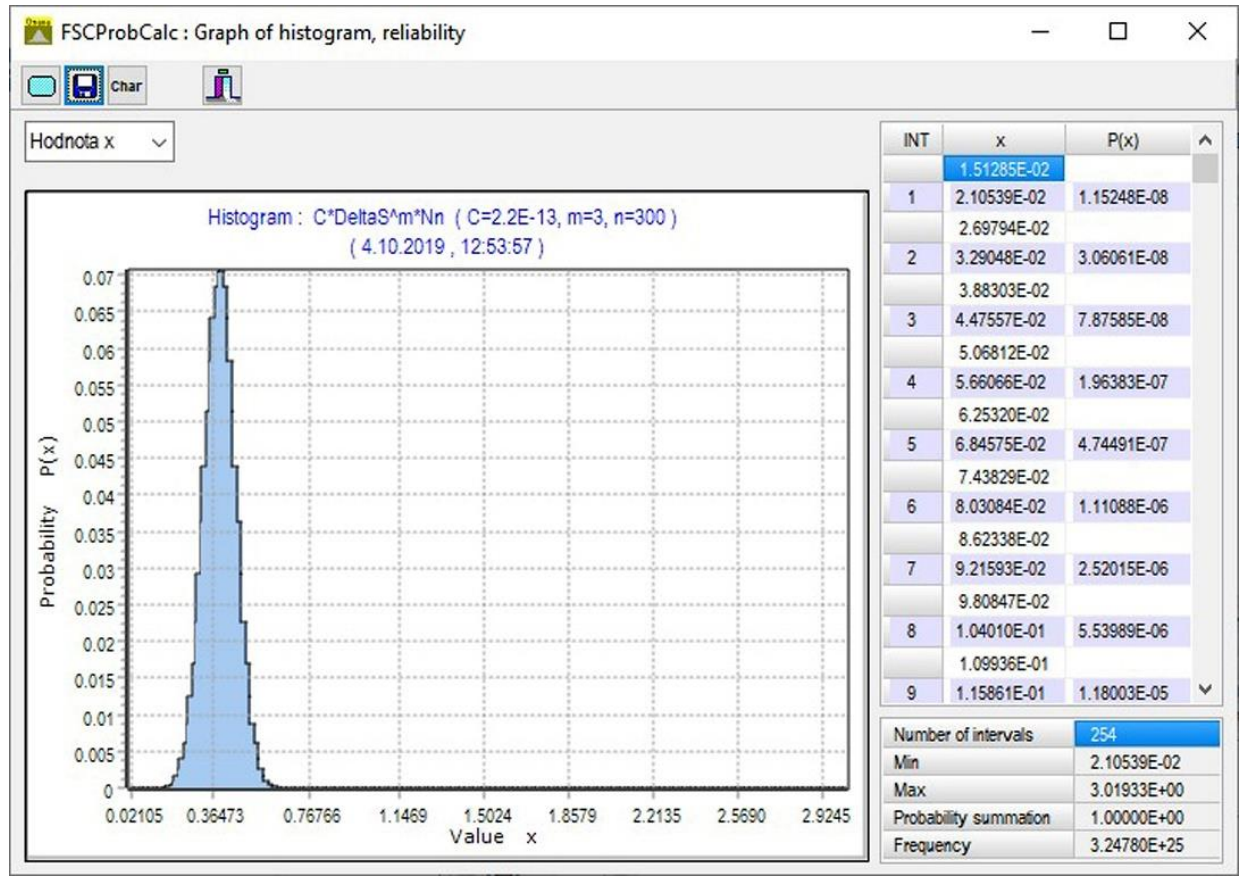

Fig. 4. FSCProbCalc desktop: Calculated histogram of accumulated load effect $E_{(N)}$ for 277 years of structural operation.

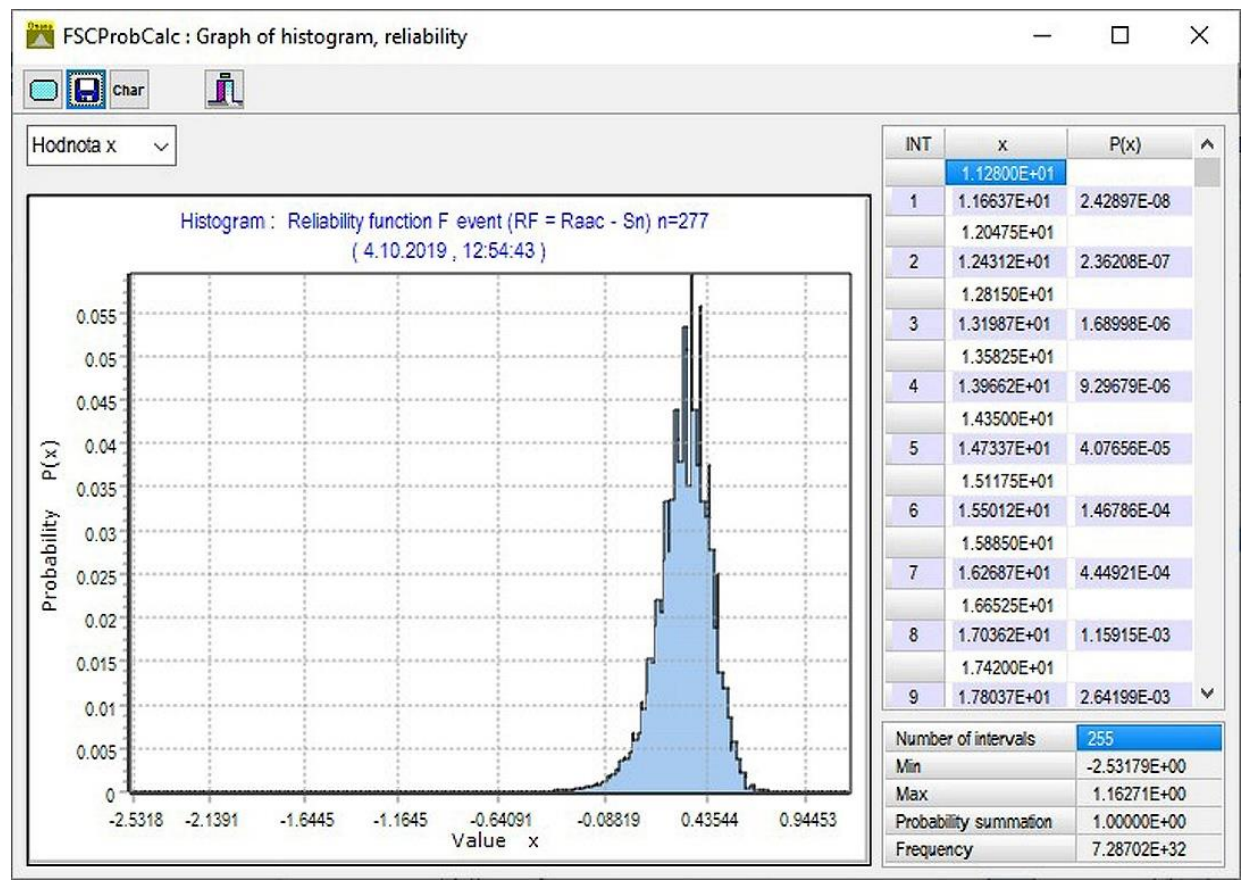

Fig. 5. FSCProbCalc desktop: Calculated histogram of safety margin $Z$ for 277 years of structural operation. 


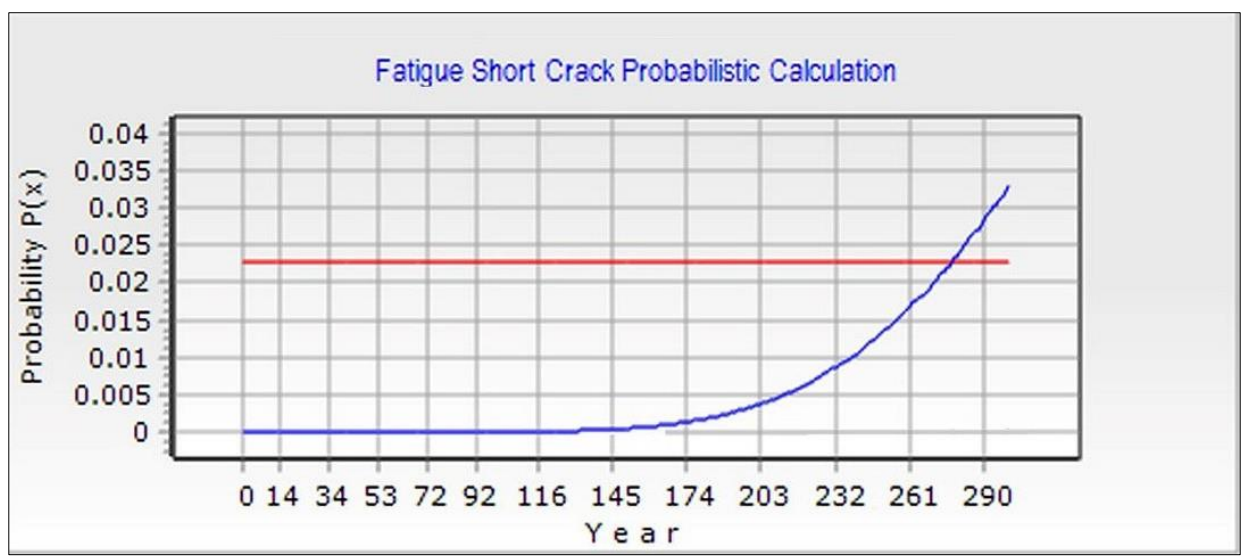

Fig. 6. FSCProbCalc desktop: Probability of failure $P_{f}$ calculation for each year of structural operation (red curve) with defined target probability (red line).

The target probability $P_{d}$ is exceeded in the $277^{\text {th }}$ year of the structural operation, which corresponds to a real lifetime of the analysed steel structure. This value is comparable to the result obtained in [20].

\section{Conclusion}

The objective of this study was to propose and evaluate a probabilistic approach for the fatigue damage analysis of an existing steel truss structure supporting an overhead crane track. To demonstrate the proposed approach, a fatigue analysis of the diagonal of the steel truss structure was presented in this paper.

The methodology for the probabilistic modeling of fatigue crack propagation in the loadbearing member of a cyclically stressed steel structure was applied in FSCProbCalc code. The resulting probability of failure was obtained for stochastic described phenomena which are related to propagation of the fatigue cracks and calculated for each year of operation of the construction. When determining the required degree of reliability, it is possible to specify the time of inspection focused on the structural fatigue damage.

The fatigue resistance of the crane can therefore be assumed to be sufficient. The fatigue damage accumulated in old structure during time of the operation is negligible in comparison with, for example, corrosion losses.

The processed methodology together with the stated application thus may substantially improve the estimation of costs spent in maintenance of cyclically loaded constructions and bridges.

This contribution has been developed as a part of the research project GACR 17-01589S "Advanced computational and probabilistic modelling of steel structures taking account fatigue damage" supported by the Czech Grant Agency and also has been completed thanks to the financial support provided to VSB-Technical University of Ostrava by the Czech Ministry of Education, Youth and Sports from the budget for conceptual development of science, research and innovations for the 2019 year. 


\section{References}

1. J. Vican, J. Gocal, J. Odrobinak, P. Kotes, Procedia Engineering 156, 507-514 (2016).

2. D. Leonetti, J. Maljaars, H. H. B. Snijder, Engineering Structures 186, 471-483 (2019).

3. N. Lu, Y. Liu, Y. Deng, International Journal of Steel Structures 19, 1, 181-192 (2019).

4. T. A. A. Oliveira, G. Gomes, F. Evangelista Jr, Engineering Analysis with Boundary Elements 104, 107-119 (2019).

5. A. Omishore, IOP Conference Series: Materials Science and Engineering 471, 10, 102037 (2019).

6. C. S. Wang, M. S. Zhai, L. Duan, Q. Wang, Advanced Steel Construction 11, 3, 305321 (2015).

7. T. P. McAllister, B. R. Ellingwood, Structural Safety 23, 4, 445-465 (2001).

8. X. W. Ye, Y. H. Su, J. P. Han, Mathematical Problems in Engineering 2014, 956473, 113 (2014).

9. K. Kotrasova, Vibroengineering Procedia 23, $72-75$ (2019).

10. E. Kormanikova, K. Kotrasova, MATEC Web of Conferences 125, 04010 (2017).

11. M. Major, I. Major, Procedia Engineering 190, 223-230 (2017).

12. V. Salajka, P. Hradil, J. Kala, Applied Mechanics and Materials 284-287, 1247-1250 (2013).

13. T. L. Anderson, Fracture Mechanics: Fundamentals and Applications (CRC Press, 2004)

14. Z. Kala, Journal of Civil Engineering and Management 21, 7, 902-911 (2015).

15. J. Kralik, Procedia Engineering 190, 472-479 (2017).

16. K. Tvrda, MATEC Web of Conferences 107, 00058 (2017).

17. Z. Kala, Engineering Structures 194, 36-45 (2019).

18. L. Zhao, X. Huang, Y. Zhang, Y. Tian, Y. Zhao, Electronics 8, 5, 515 (2019).

19. P. Paris, F. Erdogan, Journal of Basic Engineering 85, 4, 528-534 (1963).

20. P. Lehner, M. Krejsa, P. Parenica, V. Krivy, J. Brozovsky, International Journal of Fatigue 128, 105190 (2019).

21. S. Seitl, P. Miarka, P. Pokorny, S. Fintova, L. Kunz, Transactions of the VSB - Technical University of Ostrava, Civil Engineering Series 19, 2, 54-58 (2019).

22. R. Sanches, A. De Jesus, J. Correia, A. L. L. Silva, A. Fernandes, Journal of Constructional Steel Research 110, 149-162 (2015).

23. S. Seitl, P. Miarka, L. Malikova, M. Krejsa, Key Engineering Materials 5, 353-356 (2017).

24. S. Seitl, P. Miarka, Z. Kala, J. Klusak, Procedia Structural Integrity 5, 697-704 (2017). 